\title{
Evaluation of the diagnostic potential of ex vivo Raman spectroscopy in gastric cancers: fingerprint versus high wavenumber
}

\author{
Xueqian Zhou \\ Jianhua Dai \\ Yao Chen \\ Guangjie Duan \\ Yulong Liu \\ Hua Zhang \\ Hongbo Wu \\ Guiyong Peng
}




\title{
Evaluation of the diagnostic potential of ex vivo Raman spectroscopy in gastric cancers: fingerprint versus high wavenumber
}

\author{
Xueqian Zhou, ${ }^{a}$ Jianhua Dai, ${ }^{a}$ Yao Chen, ${ }^{a}$ Guangjie Duan, ${ }^{b}$ Yulong Liu, ${ }^{c}$ Hua Zhang, ${ }^{c}$ Hongbo Wu, ${ }^{a}$ and \\ Guiyong Peng ${ }^{a, *}$ \\ ${ }^{a}$ Third Military Medical University, Institute of Digestive Disease, Southwest Hospital, No. 30 Gaotanyan Street, Shapingba District, \\ Chongqing 400038, China \\ ${ }^{\text {b} T h i r d ~ M i l i t a r y ~ M e d i c a l ~ U n i v e r s i t y, ~ I n s t i t u t e ~ o f ~ P a t h o l o g y ~ a n d ~ S o u t h w e s t ~ C a n c e r ~ C e n t e r, ~ S o u t h w e s t ~ H o s p i t a l, ~ N o . ~} 30$ Gaotanyan Street, \\ Shapingba District, Chongqing 400038, China \\ ${ }^{\circ}$ Chongqing Institute of Green and Intelligent Technology, Key Laboratory of Multi-scale Manufacturing Technology, Chinese Academy of \\ Sciences, No. 266 Fangzheng Avenue, Shuitu Hi-tech Industrial Park, Shuitu Town, Beibei District, Chongqing 400714, China
}

\begin{abstract}
The aim of this study was to apply Raman spectroscopy in the high wavenumber (HW) region (2800 to $3000 \mathrm{~cm}^{-1}$ ) for ex vivo detection of gastric cancer and compare its diagnostic potential with that of the fingerprint (FP) region (800 to $1800 \mathrm{~cm}^{-1}$ ). Raman spectra were collected in the FP and $\mathrm{HW}$ regions to differentiate between normal mucosa $(n=38)$ and gastric cancer $(n=37)$. The distinctive Raman spectral differences between normal and cancer tissues are observed at 853, 879, 1157, 1319, 1338, 1448, and $2932 \mathrm{~cm}^{-1}$ and are primarily related to proteins, lipids, nucleic acids, collagen, and carotenoids in the tissue. In FP and HW Raman spectroscopy for diagnosis of gastric cancer, multivariate diagnostic algorithms based on partialleast-squares discriminant analysis, together with leave-one-sample-out cross validation, yielded diagnostic sensitivities of $94.59 \%$ and $81.08 \%$, and specificities of $86.84 \%$ and $71.05 \%$, respectively. Receiver operating characteristic analysis further confirmed that the FP region model performance is superior to that of the HW region model. Better differentiation between normal and gastric cancer tissues can be achieved using FP Raman spectroscopy and PLS-DA techniques, but the complementary natures of the FP and HW regions make both of them useful in diagnosis of gastric cancer. @ 2016 Society of Photo-Optical Instrumentation Engineers (SPIE) [DOI: 10.1117/1.JBO.21.10.105002]
\end{abstract}

Keywords: Raman spectroscopy; high wavenumber; fingerprint; gastric cancer.

Paper 160270RR received Apr. 24, 2016; accepted for publication Sep. 19, 2016; published online Oct. 7, 2016.

\section{Introduction}

Decreasing incidence and mortality rates have been reported for gastric cancer in most developed countries and also in selected developing countries over the last few decades. Still, 723,100 people died of gastric cancer, 951,600 new cases occurred worldwide in $2012,{ }^{1-3}$ and $>40 \%$ of these patients were Chinese. ${ }^{3}$ Early detection and effective treatment of gastric cancer have a great impact on the 5-year survival rates of patients, ${ }^{4}$ but unfortunately, even in Japan (which is a highly developed Asian country), difficulties occur in early detection of gastric cancer because of a shortage of skilled endoscopists and limited availability of endoscopic devices, ${ }^{5}$ not to mention the problems and possibilities of nationwide screening for gastric cancer in China, which has the largest population in the world and great imbalances in regional development. Histological examination of gastric biopsy has been widely used since the popularization of endoscopy, ${ }^{6}$ which remains the gold standard for gastric cancer diagnosis. However, gastric biopsy under direct vision suffers from several disadvantages, such as lack of nondestructive character, extension of endoscopic examination time, and a delay of several days for the diagnostic result provided to patients. Consequently, additional efforts should be focused on the development of endoscopic diagnostic techniques that are real-time, nondestructive, and objective.
First introduced by Raman in 1928, Raman spectroscopy has shown great potential in diagnosis. ${ }^{7,8}$ This technique detects an emission spectrum generated by high-frequency inelastic scattering of light resulting from the response of a molecule to incident rays. ${ }^{7}$ Each molecular motion creates its own individual bands in the Raman spectrum. ${ }^{7}$ Hence, Raman spectroscopy reveals biomolecular changes in the human body caused by malignancy and presents an opportunity for use as a promising tool for doctors in cancer diagnosis. In recent years, Raman spectroscopy has been used to diagnose different types of cancers and precancers in vivo and ex vivo, including glioma, nasopharyngeal carcinoma, Barrett's esophagus, lung cancer, breast cancer, and melanoma. $^{9-14}$ The sensitivity and specificity for diagnosis of malignancies reported in these research studies are satisfactory and encouraging. ${ }^{9-14}$

The Raman probe is sufficiently slender to freely pass through the instrument channel of an endoscope. Gastric biopsy is a routine process for patients suspected of suffering from gastric cancer, and this technique makes it easier to obtain samples compared with parenchymal viscera (e.g., brain, liver). Many doctors and optical scientists attempted to improve the diagnostic effect of Raman spectroscopy for gastrointestinal tumors both in vivo and ex vivo. ${ }^{15-19}$ However, most of these studies focus on the fingerprint (FP) Raman spectroscopy 
range of 800 to $1800 \mathrm{~cm}^{-1}$, which presents biochemical information on the backbone structures of DNA, RNA, proteins, lipids, collagen, and other biological macromolecules. In 2014, we also used the FP region of Raman spectroscopy to explore the biochemical characteristics of molecules associated with the malignant transformation of gastric mucosa. ${ }^{20}$ In our previous study, the great value of the high wavenumber (HW) region (2400 to $3800 \mathrm{~cm}^{-1}$ ) in diagnosis was not yet evaluated and discussed. ${ }^{20}$ Compared with the main advantages of the HW region, in which the intense background signal of the silica fiber-optic probe is absent and more intense tissue Raman signals are generated, it appears that the FP region provides much more information on the molecular composition and structure of tissue. $^{21-27}$ However, the background-signal-free design of the Raman probe for FP Raman spectroscopy is much more complicated and expensive, ${ }^{21}$ which could further affect development and medical application of in vivo diagnostic tools based on Raman spectroscopy. ${ }^{21}$ Therefore, it is worthwhile to comprehensively investigate the diagnostic effect of both the FP and HW regions.

In this research, we compared the diagnostic potential of FP (800 to $1800 \mathrm{~cm}^{-1}$ ) Raman spectroscopy with that of HW (2800 to $3000 \mathrm{~cm}^{-1}$ ) Raman spectroscopy in gastric cancers. The biochemical changes of molecules found in both the FP region and the HW region were thoroughly reviewed as well. Multivariate statistical techniques, including partial least-squares-discriminant analysis (PLS-DA), were used to design diagnostic algorithms for discrimination between normal mucosa and gastric cancer.

\section{Materials and Methods}

\subsection{Raman Instrumentation and Spectral Measurements}

The Raman spectra of gastric biopsy samples were recorded using a micro-Raman spectroscopy system (inVia Reflex, Renishaw, UK). A 785-nm laser (maximum output of $300 \mathrm{~mW}$, Renishaw, UK) was used as the excitation source, with a power of $10 \%$ and an integration time of $10 \mathrm{~s}$. The instrument was equipped with a Leica $50 \times$ objective. The laser power on the surface of the samples was $\sim 20 \mathrm{mw}$, and the resolution of the spectroscopy technique is $2 \mathrm{~cm}^{-1}$. To ensure that the instrument was operating properly, silicon was used to check the spectrum prior to recording. Samples were removed from the storage vial and thawed at room temperature. Each sample was placed on an aluminum holder and measured under the same conditions. Two to seven observation fields were randomly selected, according to the size of each tissue sample. The average was used to represent the Raman spectrum of the sample.

\subsection{Patients and Biopsy Sample Collections}

Biopsy samples of gastric cancer were collected from patients suspected of suffering from gastric cancer during routine upper gastrointestinal endoscopic exams at Southwest Hospital, first affiliated hospital of Third Military Medical University. Biopsy samples of normal tissue were collected from healthy volunteers in the same hospital who were free from any other diseases or treatments. Informed consent was obtained from the patients and healthy volunteers prior to sample collection. This study was approved by Southwest Hospital Ethics Committee, in agreement with the Helsinki declaration. The
Table 1 Patient characteristics.

\begin{tabular}{lcc}
\hline & Normal $(n=38)$ & Gastric cancer $(n=37)$ \\
\hline Age (years) & $43.4 \pm 11.8$ & $63.2 \pm 9.3$ \\
Gender (F/M) & $10 / 27$ \\
Biopsy location & $21 / 17$ & 10 \\
Upper third & 14 & 19 \\
Middle third & 14 & 8 \\
Lower third & 10 & 9 \\
Pathological diagnosis & & 28 \\
Diffuse type & - & \\
Intestinal type & - & \\
\hline
\end{tabular}

methods were carried out in accordance with the approved guidelines. No patients received any treatments such as chemotherapy or radiotherapy prior to sample collection. Tissue specimens were snap frozen in liquid nitrogen until analysis. After the Raman measurements were completed, the tissue samples were fixed with $10 \%$ formalin and pathologically confirmed. In all, 38 normal and 37 gastric cancer biopsy samples (each from different patients and healthy volunteers) were included in this research. The characteristics of the patients and healthy volunteers are listed in Table 1 . The histological type was categorized as diffuse type or intestinal type according to the Lauren classification. ${ }^{28}$

\subsection{Data Processing}

The Raman spectrum in the wavenumber range of 400 to $3000 \mathrm{~cm}^{-1}$ was acquired from all tissue samples to analyze and compare the diagnostic effect of FP Raman spectroscopy with that of HW Raman spectroscopy. In this research, our analyses were limited to the FP region (800 to $1800 \mathrm{~cm}^{-1}$ ) and the HW region (2800 to $3000 \mathrm{~cm}^{-1}$ ) because these two regions provide the greatest biochemical information from human tissue. ${ }^{29}$ The acquired raw Raman spectra consisted of tissue autofluorescence background and noise, therefore, a second-order Savitzky-Golay filter (window width of 7 pixels) was used to reduce the noise. Subsequently, the autofluorescence background in the noise-smoothed spectrum was subtracted using an algorithm known as adaptive iteratively reweighted penalized least squares (airPLS) that does not require any user intervention or prior information. ${ }^{30}$ To compare the spectral shapes and relative Raman band intensities between the different samples, all of the acquired Raman spectra were normalized to the integrated area under the curve (FP or HW). All data were processed in the MATLAB environment (The MathWorks, Inc., Natick, Massachusetts).

\subsection{Statistical Analysis}

Probabilistic PLS-DA, which was reported to be more effective than principal component analysis (PCA) in cell and tissue classification, was applied to evaluate the difference between normal gastric mucosa and gastric cancer. ${ }^{31,32}$ The leaveone-sample-out cross-validation method was used to confirm 
the performance of the PLS-DA diagnostic algorithm. The number of retained latent variables (LVs) was determined based on the error rate of the cross-validation curves. The receiver operating characteristic (ROC) curves were used to further compare the performance of the PLA-DA models using the two spectral data sets (FP, HW) for ex vivo diagnosis of gastric cancer.

\section{Results}

Comparison of the mean intensities of FP and HW Raman spectra with their standard deviations (SD) is shown in Fig. 1. The prominent tissue Raman peaks in the FP region are observed at 829, 853, 879, 937, 1003, 1033, 1086, 1127, $1157,1173,1207,1319,1338,1448,1526$, and $1658 \mathrm{~cm}^{-1}$. Tentative vibrational mode assignments for the human gastric tissue Raman peaks in the FP region are summarized in Table 1 of our previous work. ${ }^{20}$ Moreover, intense Raman peaks are also found in the HW region, e.g., $2849 \mathrm{~cm}^{-1}$ (symmetric $\mathrm{CH}_{2}$ stretching of lipids), $2875 \mathrm{~cm}^{-1}\left(\mathrm{CH}_{2}\right.$ symmetric

(a)
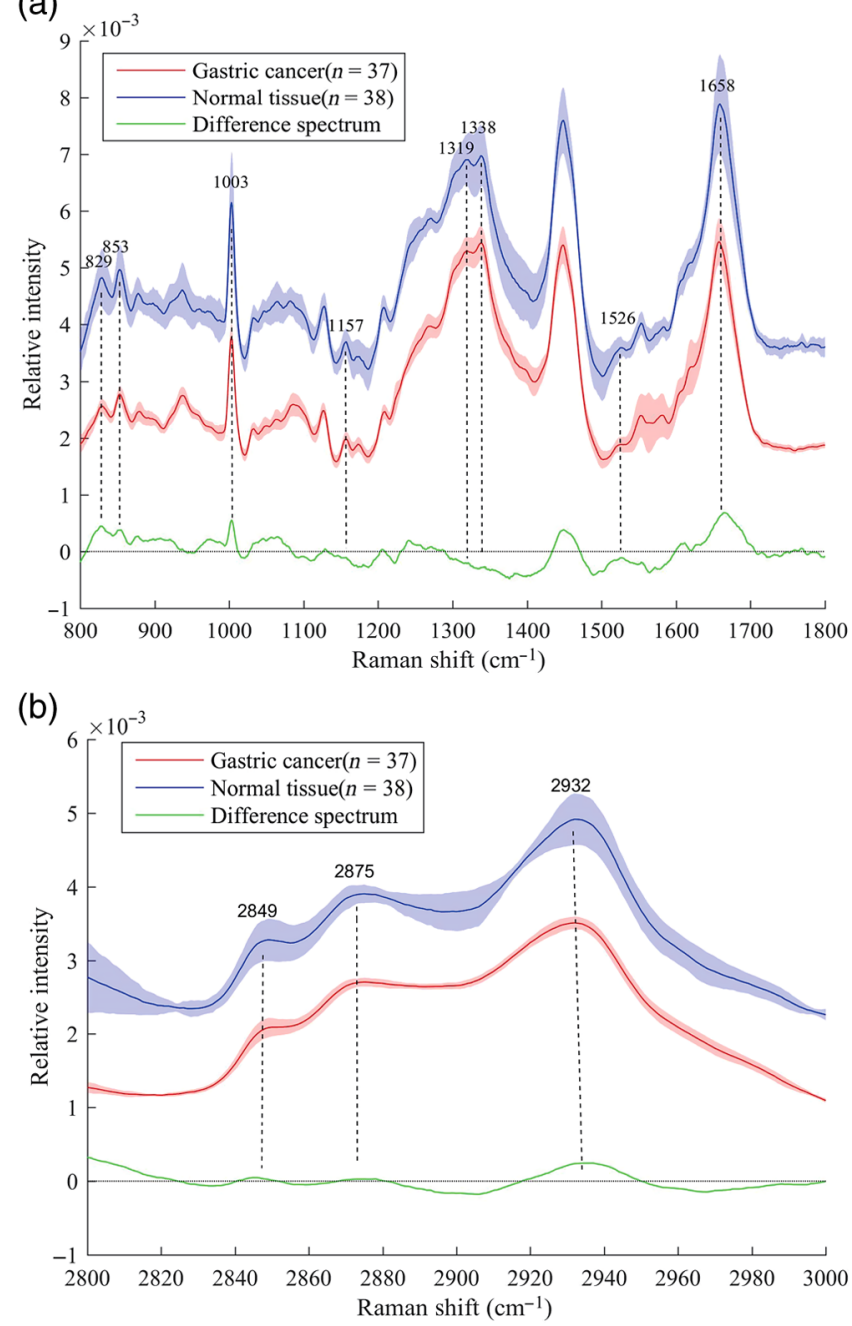

Fig. 1 (a) Mean FP Raman spectra of 38 normal and 37 gastric cancer tissues. (b) Mean HW Raman spectra of 38 normal and 37 gastric cancer tissues. The Raman spectra for normal tissues (blue line) are shifted for better visualization. The red-shaded and blueshaded areas represent the respective SD of the means. Difference spectra (normal-gastric cancer) calculated from the average Raman spectra between normal tissue and gastric cancer are shown with a green line, and the main Raman peaks are marked. stretch of lipids and $\mathrm{CH}_{2}$ asymmetric stretch of lipids and proteins), and $2932 \mathrm{~cm}^{-1}\left(\mathrm{CH}_{3}\right.$ stretching of proteins). ${ }^{21,33}$ Independent-sample $t$-tests showed that Raman peak intensities are notably higher for gastric cancer at $1157 \mathrm{~cm}^{-1}$ (polyene chain of carotenoids), $1173 \mathrm{~cm}^{-1}[\delta \mathrm{C}-\mathrm{H}$ in-plane bending of phenylalanine, tyrosine (protein)], 1319 and $1338 \mathrm{~cm}^{-1}$ $\left(v \mathrm{CH}_{2} \mathrm{CH}_{3}\right.$ and $\delta \mathrm{CH}_{2} \mathrm{CH}_{3}$ of proteins and nucleic acids), and $1526 \mathrm{~cm}^{-1} \quad(v \mathrm{C}-\mathrm{C}$ of carotenoids), but notably lower differences are significant at $829 \mathrm{~cm}^{-1}$ (vas $\mathrm{PO}_{2}^{-}$of nucleic acid), $853 \mathrm{~cm}^{-1}$ [vC $-\mathrm{C}$ of proline (collagen) $], 879 \mathrm{~cm}^{-1}$ [ $v \mathrm{C}-\mathrm{C}$ hydroxyproline (collagen) $], 1003 \mathrm{~cm}^{-1}$ (vring breathing of phenylalanine $), 1033 \mathrm{~cm}^{-1}(\delta \mathrm{C}-\mathrm{H}$ plane bending of aromatic compounds), $1448 \mathrm{~cm}^{-1}\left(\delta \mathrm{CH}_{2}\right.$ of proteins and lipids), $1658 \mathrm{~cm}^{-1}$ [amino compounds I $\left(\alpha\right.$-helix)], and $2932 \mathrm{~cm}^{-1}$ $\left(\mathrm{CH}_{3}\right.$ stretching of proteins). To better reflect the differences in the main Raman peaks between normal tissue and gastric cancer, a comparison of the mean intensities and SDs is shown in Fig. 2.

The PLS-DA with leave-one-sample-out cross validation was applied to generate diagnostic algorithms for differentiation between normal tissue and gastric cancer. As shown in Fig. 3, six LVs were found to be the optimal number of retained

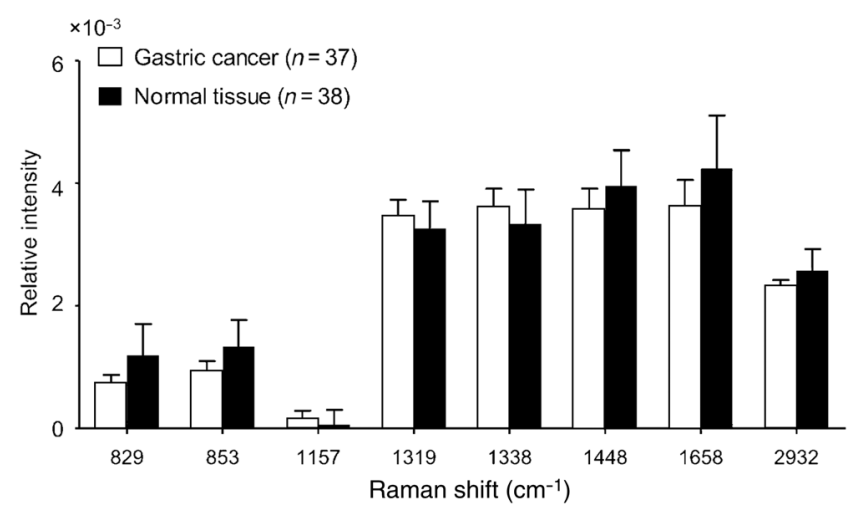

Fig. 2 Comparison of the mean intensities and SD of selected Raman peaks showing significant differences in independent-sample $t$-tests between normal tissues and gastric cancer.

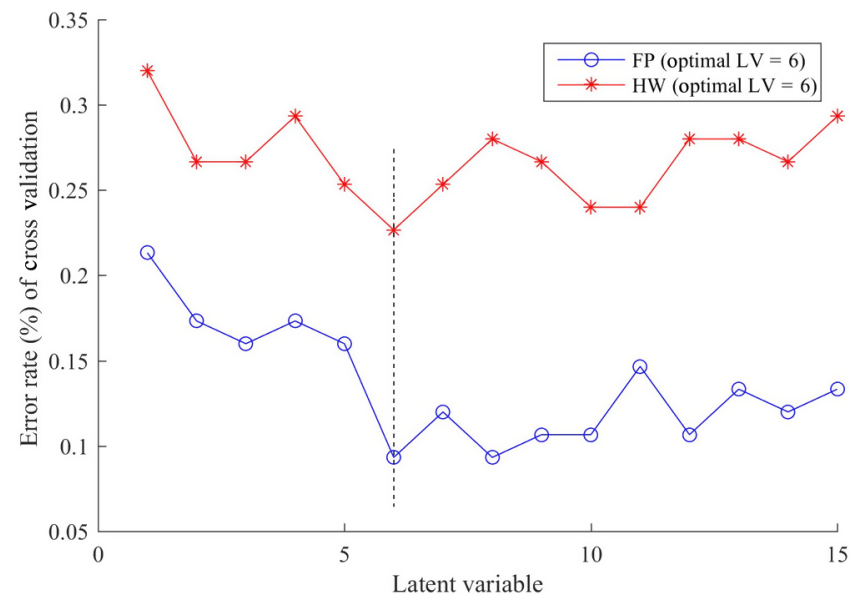

Fig. 3 Relationship between the numbers of PLS factors-LVs and the error rate of cross validation for correct diagnosis of normal mucosa and gastric cancer. The error rate shows that the optimal number of retained components for PLS-DA modeling of both the FP and HW regions is 6 LVs. 

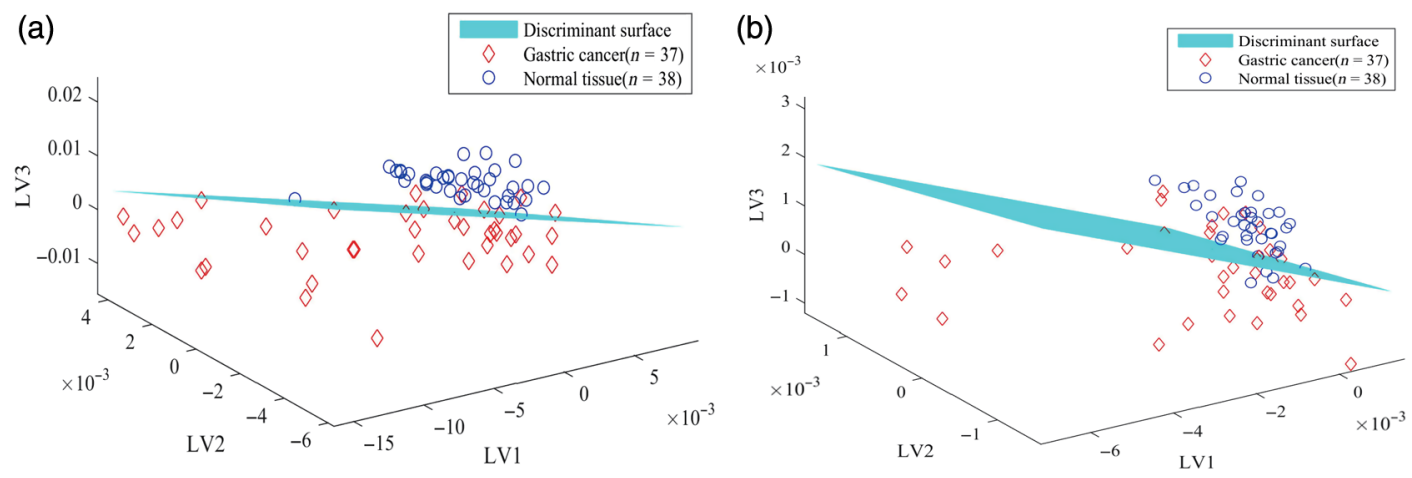

Fig. 4 (a) Three-dimensional scatter plots spanned by the three LVs (LV1, LV2, and LV3) of the FP region demonstrating the discrimination between normal mucosa and gastric cancer. (b) Threedimensional scatter plots spanned by the three LVs (LV1, LV2, and LV3) of the HW region demonstrating the discrimination between normal mucosa and gastric cancer.

components for PLS-DA modeling of both the FP region and HW region as defined by the minimum error rate of cross validation. To demonstrate the use of LVs for diagnosis and the intrinsic clustering of normal mucosa and gastric cancer, two three-dimensional (3-D) scatterplots are presented in Fig. 4 using the three LVs (LV1, LV2, and LV3) of the FP and HW regions, respectively. The $L V$ points are divided into two distinct

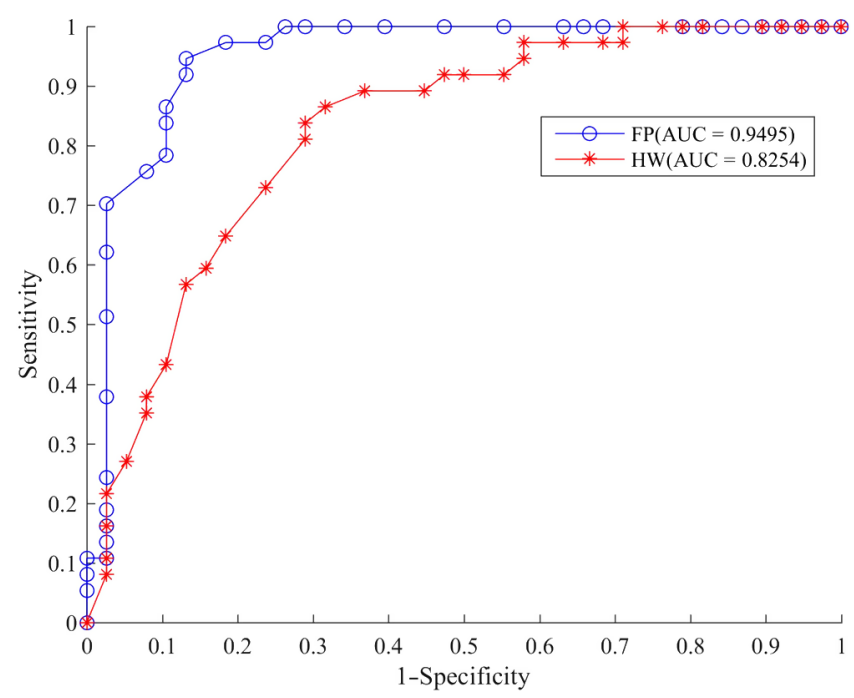

Fig. 5 ROC curves of diagnostic performance for the FP and HW Raman spectra for gastric cancer diagnosis via Raman spectroscopy and PLS-DA together with the leave-one-sample-out cross-validation method. The integrated areas under the ROC curves are 0.9495 and 0.8254 for the FP and HW Raman spectra, respectively. groups, suggesting that both FP and HW Raman spectroscopy have potential for use as a clinical diagnosis tool for gastric cancer. The developed PLS-DA algorithm of the FP region achieves diagnostic sensitivities of $94.59 \%$, specificities of $86.84 \%$, and accuracies of $90.67 \%$. The HW modeling achieves diagnostic sensitivities of $83.78 \%$, specificities of $71.05 \%$, and accuracies of $77.33 \%$. The ROC curves (Fig. 5) are also generated for the two spectral datasets (FP, HW) to further evaluate the diagnostic performance. The integration areas under the ROC curves for FP modeling are 0.9495, and for HW modeling, the number is 0.8254 . The diagnostic performance comparisons (i.e., sensitivities, specificities, accuracies, and AUCs) of the FP and HW PLS-DA modeling are summarized in Table 2.

\section{Discussion}

Raman spectroscopy facilitates the collection of specific molecular information for tissue diagnosis and characterization in a nondestructive manner. This distinctive vibrational spectroscopic technique allows researchers to study the tissue in its natural condition without the need for tissue preparation. ${ }^{18}$ Currently, Raman studies of gastric cancer have evaluated Raman spectra mostly in the FP region. ${ }^{16-19,34}$ Only a few articles have reported Raman measurements from both the FP and HW regions, ${ }^{34}$ but multivariate statistical analysis was not performed in these studies to evaluate the diagnostic potential of the FP and HW regions. In this work, we compared the diagnostic potential of FP Raman spectroscopy in gastric cancers with that of HW Raman spectroscopy and investigated the biochemical information contained in both spectral regions for the purpose of tissue characterization.

When normal tissues experience the process of malignant disease initiation, its structural conformation and relative

Table 2 Comparison of diagnostic performance of different Raman spectral regions (FP, HW) for differentiation of gastric cancer from normal mucosa.

\begin{tabular}{lccccc}
$\begin{array}{l}\text { Raman } \\
\text { spectroscopic } \\
\text { modalities }\end{array}$ & Retained LVs & Sensitivity (\%) & Specificity (\%) & Accuracy (\%) & ROC (AUC) \\
\hline FP & 6 & $94.59(35 / 37)$ & $86.84(33 / 38)$ & $90.67(68 / 75)$ & 0.9495 \\
HW & 6 & $83.78(31 / 37)$ & $71.05(27 / 38)$ & $77.33(58 / 75)$ & 0.8254 \\
\hline
\end{tabular}

Abbreviations: FP, fingerprint, HW, high wavenumber, LVs, latent variables, ROC, receiver operating characteristic, AUC, area under curve. 
content of macromolecules are altered. The main advantage of using the FP region is that it provides highly specific information on proteins, lipids, and nucleic acids associated with malignancy. ${ }^{31}$ For instance, in a previous study, we reported that the peak at $1526 \mathrm{~cm}^{-1}$ was significantly stronger in gastric cancer tissue than normal tissue $(p<0.05)$, indicating that the carotenoid content in cancer is increased. ${ }^{20}$ In this work, another peak at $1157 \mathrm{~cm}^{-1}$ (polyene chain of carotenoids) was also found to be significantly stronger in gastric cancer tissue $(p<0.05)$. These observations provide further evidence for our assumptions that cancer cells probably evolve an enhanced ability to resist damage because of the antioxidant function of carotenoid. ${ }^{20}$ In addition, the peak at $853 \mathrm{~cm}^{-1}[v \mathrm{C}-\mathrm{C}$ of proline (collagen)] was observed to be lower for gastric cancer tissue. A possible reason for this result is that cancer cells synthesize and secrete matrix metalloproteinase that degrades matrix proteins such as collagen to assist cancer metastasis. ${ }^{20}$ Huang et al. ${ }^{35}$ also observed a decrease of collagen in gastric cancer in vivo using a novel image-guided Raman endoscopy technique. In addition, the protein and nucleic acid Raman signals at 1319 and $1338 \mathrm{~cm}^{-1}$, which most likely arise from the chromosomes in the nucleus, have been found to be significantly higher for gastric cancer tissues compared with normal tissue. This observation might be explained by an increase in nuclear chromatin and excessive DNA replication during malignant transformation. Therefore, the distinct differences in the FP region between normal tissues and gastric cancer reinforce the hypothesis that the FP region can be used to interpret the biochemical transformation of molecules associated with gastric mucosa malignancy.

However, the HW region (2800 to $3000 \mathrm{~cm}^{-1}$ ) appears to contain less biochemical information than the FP region. Raman peaks at 2849, 2875, and $2932 \mathrm{~cm}^{-1}$ are observed in the HW region. The protein signal at $2932 \mathrm{~cm}^{-1}$ was significantly stronger in normal tissue than in the gastric cancer tissue $(p<0.05)$. The other two peaks showed no significant difference. However, this result does not mean that the HW region is less important for diagnosis. The HW region contains information related to $\mathrm{CH}_{3}$ stretching of proteins, which are complementary to the properties of the FP region. ${ }^{36}$ In addition, the biochemical information provided by the HW region is sufficient for cancer diagnosis. ${ }^{23}$ Nijssen et al. reported that a linear discriminant analysis (LDA)-based tissue classification model using the HW region (2800 to $3125 \mathrm{~cm}^{-1}$ ) discriminated between basal cell carcinoma (BCC) and normal skin with high accuracy. ${ }^{23}$

We further used PLS-DA with leave-one-sample-out cross validation to generate diagnostic algorithms for gastric cancer using the FP region and the HW region. The PLS-DA modeling of the FP region, which achieved diagnostic accuracies of $90.67 \%(68 / 75)$ [sensitivities of $94.59 \%(35 / 37)$ and specificities of $86.84 \%(33 / 38)]$ performed better than the HW region modeling. The HW modeling misclassified 17 samples out of the total 75 samples, but the FP modeling reduced the number of misclassified samples to 7. Similar results were also observed in cervical precancers that were investigated using simultaneous FP (800 to $1800 \mathrm{~cm}^{-1}$ ) and HW (3100 to $3700 \mathrm{~cm}^{-1}$ ) confocal Raman spectroscopy. ${ }^{31}$ The ROC curves (Fig. 5) further validate the better performance and robustness of the FP region modeling. Fortunately, compared with the accuracy of gastric biopsy under direct vision, which was reported to vary from $50 \%$ to $96 \%{ }^{6}$ the performances of both FP modeling and the HW modeling are satisfactory for diagnosis of gastric cancer. Furthermore, we also performed the joint analysis of these two regions, but the diagnostic power is the same with the FP modeling. The FP + HW modeling also achieves diagnostic sensitivities of $94.59 \%$, specificities of $86.84 \%$, and accuracies of $90.67 \%$.

Although our work showed that FP region modeling provided diagnostic advantages over HW region modeling, the development of both FP region and HW region diagnostic technologies should be encouraged because the HW region contains information related to lipids, proteins, and water bands that does not show up in the FP region. ${ }^{36}$ Another reason is that the great difference in composition of human tissue (e.g., brain and bone) makes it worthwhile to find a suitable region of Raman spectroscopy for use in building diagnostic algorithms. In addition, it has been reported that diagnostic algorithms developed ex vivo might not be directly used for in vivo applications in the colon. ${ }^{37}$ The performance comparison of the FP and the HW diagnostic algorithms developed for gastric cancer in vivo is not complete, therefore, further investigations are needed to determine whether the performance of the FP region modeling is still better than the HW region modeling in vivo. Another limitation of this study was the constraint on experimental conditions which means that the sample size of our study might not be sufficiently large to provide better PLS-DA modeling of both the FP region and HW region. We plan to enlarge the sample size to improve the diagnostic algorithms in our future studies. Currently, we are working on prototype spectrometers with a Raman probe that is compatible with the instrument channel of the endoscope for in vivo applications. The ultimate goal of our work is to develop a Raman spectroscopy diagnosis system that can be used as a diagnostic tool during screening for gastric cancer and will also provide an accurate gastrointestinal tumor margin assessment during endoscopic submucosal dissection. It is difficult to predict whether Raman spectroscopy can replace histopathology, but we do believe that this approach will provide good clinical tools for endoscopists in the future.

\section{Conclusions}

We compared the diagnostic potential of FP Raman spectroscopy in gastric cancers with that of HW Raman spectroscopy ex vivo and uncovered the biochemical information contained in both spectral regions for the purpose of tissue characterization. Better differentiation between normal and gastric cancer tissues can be achieved using FP Raman spectroscopy and PLS-DA techniques. However, the HW region contains complementary spectral information that might have a positive effect for gastric cancer diagnosis. We expect that both FP Raman spectroscopy and HW Raman spectroscopy will become useful clinical diagnostic tools for the early detection of gastric cancer in the future.

\section{Acknowledgments}

This work was supported by the National Natural Science Foundation of China (Grant No. 81272580) and Chongqing Science \& Technology Commission (Grant No. cstc2015shmszx 10017). The funder had no role in study design, data collection and analysis, decision to publish, or preparation of the manuscript. 


\section{References}

1. M. Malvezzi et al., "An age-period-cohort analysis of gastric cancer mortality from 1950 to 2007 in Europe," Ann. Epidemiol. 20(12), 898-905 (2010)

2. P. Guo et al., "Trends in cancer mortality in China: an update," Ann. Oncol. 23(4), 2755-2762 (2012).

3. J. Ferlay et al., "Cancer incidence and mortality worldwide: sources, methods and major patterns in GLOBOCAN 2012," Int. J. Cancer 136(5), E359-E386 (2015).

4. Y. Isobe et al., "Gastric cancer treatment in Japan: 2008 annual report of the JGCA nationwide registry," Gastric Cancer 14(4), 301-316 (2011).

5. W. K. Leung et al., "Screening for gastric cancer in Asia: current evidence and practice," Lancet Oncol. 9(3), 279-287 (2008).

6. A. R. W. Hatfield et al., "Importance of the site of endoscopic gastric biopsy in ulcerating lesions of the stomach," Gut 16(11), 884-886 (1975).

7. C. V. Raman and K. S. Krishnan, "A new type of secondary radiation," Nature 121(3048), 501-502 (1928).

8. I. Nabiev, I. Chourpa, and M. Manfait, "Applications of Raman and surface-enhanced Raman scattering spectroscopy in medicine," J. Raman Spectrosc. 25, 13-23 (1994).

9. M. Jermyn et al., "Intraoperative brain cancer detection with Raman spectroscopy in humans," Sci. Trans. Med. 7(274), 274ra219274ra19 (2015).

10. Y. Li et al., "Micro-Raman spectroscopy study of cancerous and normal nasopharyngeal tissues," J. Biomed. Opt. 18(2), 027003 (2013).

11. C. Kendall et al., "Raman spectroscopy, a potential tool for the objective identification and classification of neoplasia in Barrett's oesophagus," J. Pathol. 200(5), 602-609 (2003).

12. Z. Huang et al., "Near-infrared Raman spectroscopy for optical diagnosis of lung cancer," Int. J. Cancer. 107(6), 1047-1052 (2003).

13. A. S. Haka et al., "Diagnosing breast cancer by using Raman spectroscopy," Proc. Natl. Acad. Sci. U. S. A. 102(35), 12371-12376 (2005).

14. M. Gniadecka et al., "Melanoma diagnosis by Raman spectroscopy and neural networks: structure alterations in proteins and lipids in intact cancer tissue," J. Invest. Dermatol. 122(2), 443-449 (2004).

15. I. Latka et al., "Fiber optic probes for linear and nonlinear Raman applications-Current trends and future development," Laser Photonics Rev. 7(5), 698-731 (2013).

16. S. K. Teh et al., "Diagnosis of gastric cancer using near-infrared Raman spectroscopy and classification and regression tree techniques," J. Biomed. Opt. 13(3), 034013 (2008).

17. T. Kawabata et al., "Optical diagnosis of gastric cancer using nearinfrared multichannel Raman spectroscopy with a 1064-nm excitation wavelength," J. Gastroenterol. 43(4), 283-290 (2008).

18. M. S. Bergholt et al., "Raman endoscopy for in vivo differentiation between benign and malignant ulcers in the stomach," Analyst 135(12), 3162-3168 (2010).

19. S. Luo et al., "Discrimination of premalignant lesions and cancer tissues from normal gastric tissues using Raman spectroscopy," J. Biomed. Opt. 18(6), 067004 (2013).

20. Y. Chen et al., "Raman spectroscopy analysis of the biochemical characteristics of molecules associated with the malignant transformation of gastric mucosa," PLoS One 9(4), e93906 (2014).

21. L. F. Santos et al., "Fiber-optic probes for in vivo Raman spectroscopy in the high-wavenumber region," Anal. Chem. 77(20), 6747-6752 (2005).

22. S. Koljenovic et al., "Tissue characterization using high wave number Raman spectroscopy," J. Biomed. Opt. 10(3), 031116 (2005).

23. A. Nijssen et al., "Discriminating basal cell carcinoma from perilesional skin using high wave-number Raman spectroscopy," J. Biomed. Opt. 12(3), 034004 (2007).

24. N. S. Eikje et al., "Fiber optic near-infrared Raman spectroscopy for clinical noninvasive determination of water content in diseased skin and assessment of cutaneous edema," J. Biomed. Opt. 10(1), 014013 (2005).

25. A. H. Chau et al., "Fingerprint and high-wavenumber Raman spectroscopy in a human-swine coronary xenograft in vivo," J. Biomed. Opt. 13(4), 040501 (2008).

26. J. Mo et al., "High wavenumber Raman spectroscopy for in vivo detection of cervical dysplasia," Anal. Chem. 81(21), 8908-8915 (2009).
27. A. F. García-Flores et al., "High-wavenumber FT-Raman spectroscopy for in vivo and ex vivo measurements of breast cancer," Theory Chem. Acc. 130(4-6), 1231-1238 (2011).

28. P. Lauren, "The two histological main types of gastric carcinoma: diffuse and so-called intestinal-type carcinoma, An attempt at a histo-clinical classification," Acta Pathol. Microbiol. Scand. 64, 3149 (1965).

29. A. C. S. Talari et al., "Raman spectroscopy of biological tissues," Appl. Spectrosc. Rev. 50(1), 46-111 (2014).

30. Z. M. Zhang, S. Chen, and Y. Z. Liang, "Baseline correction using adaptive iteratively reweighted penalized least squares," Analyst 135(5), 1138-1146 (2010).

31. S. Duraipandian et al., "Simultaneous fingerprint and high-wavenumber confocal Raman spectroscopy enhances early detection of cervical precancer in vivo," Anal. Chem. 84(14), 5913-5919 (2012).

32. M. Hedegaard et al., "Discriminating isogenic cancer cells and identifying altered unsaturated fatty acid content as associated with metastasis status, using k-means clustering and partial least squares-discriminant analysis of Raman maps," Anal. Chem. 82(7), 2797-2802 (2010).

33. M. Jastrzebska et al., "Dimethyl suberimidate cross-linked pericardium tissue: Raman spectroscopic and atomic force microscopy investigations," J. Mol. Struct. 744-747, 789-795 (2005).

34. X.-F. Ling et al., "Investigation of normal and malignant tissue samples from the human stomach using Fourier transform Raman spectroscopy," Appl. Spectrosc. 56(5), 570-573 (2002).

35. Z. Huang et al., "In vivo detection of epithelial neoplasia in the stomach using image-guided Raman endoscopy," Biosens. Bioelectron. 26(2), 383-389 (2010).

36. M. S. Bergholt et al., "Simultaneous fingerprint and high-wavenumber fiber-optic Raman spectroscopy enhances real-time in vivo diagnosis of adenomatous polyps during colonoscopy," J. Biophotonics 8(9), 1-10 (2015).

37. A. Molckovsky et al., "Diagnostic potential of near-infrared Raman spectroscopy in the colon: differentiating adenomatous from hyperplastic polyps," Gastrointest. Endosc. 57(3), 396-402 (2003).

Xueqian Zhou received his BS degree from Hubei Medical College in 2008 and his MD degree from the Third Military Medical University in 2016. He is a PhD student in the Department of Gastroenterology, Southwest Hospital of Third Military Medical University, Chongqing, China. His current research interests include diagnosis of digestive diseases and clinic application of Raman spectroscopy.

Jianhua Dai received her MD degree from the Third Military Medical University, China, in 2009. Currently, she is an attending physician at Southwest Hospital (The First Affiliated Hospital of Third Military Medical University), where she is pursuing her doctor's degree. Her working and research field is diagnosis and treatment of digestive diseases.

Yao Chen received her BS degree from Chongqing Medical University in 2011 and her MD degree from the Third Military Medical University in 2014. Then she became a resident at Southwest Hospital. She majored in diagnosis and treatment of gastrointestinal diseases through gastrointestinal endoscopy. Her current research mainly focused on Raman spectroscopy of gastrointestinal tumors.

Guangjie Duan received his $\mathrm{PhD}$ in medicine from the Third Military Medical University in 2010. He is a clinical pathologist working in the Institute of Pathology of Southwest Hospital. $\mathrm{He}$ is the author of more than 20 publications in pathology of tumor and inflammation. His current research interests include the pathological diagnosis and molecular study of disease of digestive and urologic system.

Yulong Liu is a professor and director of the Research Center of Intelligent Equipment and Instrumentation at Chongqing Institute of Green and Intelligent Technology, Chinese Academy of Sciences. $\mathrm{He}$ is the author of more than 180 publications. He was president of the 7th Committee on Light Scattering Branch of Chinese Physical Society. Currently, his main research interests are Raman spectroscopy and Brillouin scattering technology as well as instrumentation development. 
Hua Zhang received her MD degree in chemistry from Tianjin University in 2012. She is a research assistant of the Research Center of Intelligent Equipment and Instrumentation at Chongqing Institute of Green and Intelligent Technology, Chinese Academy of Sciences. Her research concerns nanomaterials and their applications in surface enhanced Raman scattering (SERS). She has published 14 papers in international journals.

Hongbo Wu received his $\mathrm{BS}$ and $\mathrm{MD}$ degrees in clinical medicine from the Third Military Medical University. His research aims to prevent esophageal stricture after endoscopic submucosal dissection and clinic application of Raman spectroscopy.

Guiyong Peng received his BS and MD degrees from the Third Military Medical University. $\mathrm{He}$ is a professor and director of the Digestive Endoscopy Center at Southwest Hospital of Third Military Medical University, Chongqing, China. He is the author of more than 130 publications. Currently, he is on the Standing Committee of Chinese Digestive Endoscopy Society. His professional expertise lies in the diagnosis and treatment of gastrointestinal cancer through endoscopy. 